Revue d'histoire de l'Amérique française

FE REVUE D.HISTOIRE DE L'AMÉRIQUE FRANÇAISE

\title{
Études récentes sur l'histoire culturelle du pays des Illinois, 1930-1955
}

\section{John Francis McDermott}

Volume 9, numéro 4, mars 1956

URI : https://id.erudit.org/iderudit/301789ar

DOI : https://doi.org/10.7202/301789ar

Aller au sommaire du numéro

\section{Éditeur(s)}

Institut d'histoire de l'Amérique française

\section{ISSN}

0035-2357 (imprimé)

1492-1383 (numérique)

Découvrir la revue

\section{Citer cet article}

McDermott, J. F. (1956). Études récentes sur l'histoire culturelle du pays des Illinois, 1930-1955. Revue d'histoire de l'Amérique française, 9(4), 512-522. https://doi.org/10.7202/301789ar d'utilisation que vous pouvez consulter en ligne. 


\section{ÉTUDES RÉCENTES SUR L'HISTOIRE CULTURELLE DU PAYS DES ILLINOIS,}

1930-1955

De toutes les régions coloniales françaises en Amérique du Nord, le vieux pays des Illinois demeura la moins peuplée. Loin de Montréal et de Québec, et presque aussi éloignés de la Louisiane, les cinq villages du Mississipi - Cahokia, Kaskaskia, Prairie-du-Rocher, Saint-Philippe et Sainte-Anne-de-Fort Chartres - ne dépassèrent jamais en population plus de deux mille cinq cents Français. Même avec Vincennes et Ouiatenon sur la Ouabache, près de deux cents milles à l'est (deux postes jamais officiellement compris dans le pays des Illinois), et, dans la dernière partie du dix-huitième siècle, Sainte-Geneviève, SaintLouis, Saint-Charles et autres petites localités du Missouri, tout cet ensemble, disons-nous, atteint tout au plus les cinq mille âmes. Et ce petit nombre fut bientôt submergé par les Américains qui commencèrent à émigrer vers la partie est du pays des Illinois, après la remise du fort Chartres aux Britanniques en 1765. Les mêmes envahisseurs se déversèrent outre Mississipi, en Haute-Louisiane, pendant la décennie qui précéda l'achat de la Louisiane.

Néanmoins ces quelques milliers de Français du Canada, de la Louisiane, et de la France même, explorèrent tout le vaste bassin du Mississipi et de ses tributaires; ils y ont fait le commerce et au moins l'un de leurs établissements est devenu une grande ville. De savantes recherches, durant les derniers vingtcinq ans, sur l'histoire culturelle du pays des Illinois, témoignent de la valeur durable des entreprises de ces pionniers. Ils ont forcé l'attention des chercheurs. Etudes sur le folklore et la langue française, nouvelle analyse des premiers voyages, nouvelles publications de documents officiels, examen et reprises des monographies des premiers établissements, collections de tableaux et 
autres objets d'art, essais biographiques, contribuent à nous rendre plus familière l'histoire des habitants du pays des Illinois et de leur façon de vivre. Enfin, les recherches progressives de l'heure et celles qui devraient les suivre, démontreront tout ce qui reste inexploré en l'histoire culturelle de la région.

A aucune époque, sans doute, ce mince peuplement n'a-t-il produit quelque œuvre littéraire au sens strict du mot. Peutêtre pourrions-nous excepter un poème comme la Vraie Histoire, œuvre du sulpicien Pierre Huet de la Valinière, où ce dernier décrit les malheurs et les persécutions endurés par lui aux Illinois. Mais encore ce poème fut-il imprimé à Albany (1792). Mais tout peuple a sa littérature folklorique. Trois ouvrages ont ajouté valablement à notre connaissance du folklore de cette région. Ward Dorrance, dans The Survival of French in the Old District of Ste. Genevieve, (University of Missouri Studies, 1935) a publié un certain nombre de contes et de chansons du terroir. Deux ans après parut la compilation beaucoup plus considérable de Joseph-Médard Carrière: Tales from the French Folklore of Missouri (Northwestern University Press, 1937), matériaux recueillis dans les districts de Sainte-Geneviève et des Vieilles-Mines et qui sont, en l'espèce, sur la région, l'étude la plus complète et la mieux faite. En 1946, Cecelia-R. Berry a publié un volume de Folk Songs of Old Vincennes, paroles et musique.

Dans sa monographie plus haut citée, Dorrance a étudié le français du Missouri. Il y a inclus un glossaire de cinquante pages du français parlé en la région. Outre une dissertation sur le sujet et un glossaire très développé de la langue vivante en son recueil de Contes, Carrière a publié, en 1939, dans l'American Speech (14: 109-19), une étude sur le «Creole Dialect in Missouri »; deux ans plus tard, il en publiait une autre, dans la French Review, sur "The Phonology of Missouri-French: An Historical Study » (14:410-15, 510-15). Mon propre Glossary of Mississippi Valley French, 1673-1850 (Washington University Studies, 1941) est un relevé des variations morphologiques subies 
par cette langue en regard du français moderne. Tout autant qu'à l'histoire linguistique, on doit considérer cette étude comme une contribution à l'histoire sociale.

Naturellement la littérature de voyage reste de première importance pour qui étudie cette époque des débuts. Aussi la bibliographie des French Travellers in the United States, 17651932) par Frank Monaghan (New York Public Library, 1935) est-elle d'une grande utilité. On pourrait, à notre point de vue, souhaiter qu'il eût remonté beaucoup plus haut dans le passé; mais sa bibliographie n'en demeure pas moins d'un constant usage, car plusieurs de ces voyageurs ont décrit le pays des Illinois. Deux autres bibliographies donnent beaucoup à penser à qui étudie notre région: dans ses American Diaries (University of California Press, 1945), William Matthews catalogue peu de ces écrits qui aient pour auteurs des Français; mais plusieurs contiennent des commentaires sur les établissements français. La compilation plutôt dénuée de sens critique de Perry Rader, South of Forty (University of Oklahoma Press, 1947) contient aussi d'utiles références.

Parmi les spécialistes en littérature de voyage, comptons, au nombre des plus actifs, Jean Delanglez, s.j. Ce chercheur a repris l'étude des relations des premiers explorateurs: La Salle, Hennepin, Dablon, Cadillac, Tonty, Marquette, Joliette. Plusieurs des travaux du jésuite ont paru dans Mid-America pendant les années 40 et une partie de ceux-ci a été mise en volumes. Le lieutenant de La Salle, l'homme à la main de fer, a fait aussi l'objet d'une étude que nous devons à Edmund-R. Murphy. Son Henry de Tonty: Fur Trader of the Mississippi, a été publié par l'Institut français de Washington en 1941. Norman Caldwell s'est chargé, pour sa part, d'un autre explorateur fort actif: "Charles Juchereau de St. Denys: A French Pionner in the Mississippi Valley » (Mississippi Valley Historical Review (28: 563-80, 1942). Fleur de Lys and Calumet: The Pénicaut Narrative (traduit et étudié par Richebourg-Gaillard McWilliams, Louisiana State University Press, 1953), donne un aperçu de première main du pays des Illinois à ses tout premiers débuts. Si nous remontons à la fin du dix-huitième siècle et davantage vers 
le haut-Missouri (ma vallée du Mississipi s'étend des Alléghanys aux Rocheuses), nous trouvons, pour cette région, la superbe édition de la Tabeau's Narrative of Loisel's Expedition to the Upper Missouri d'Annie-Héloöse Abel. Ce récit d'un témoin oculaire qui a parcouru le Missouri supérieur, y a connu les tribus indiennes, leur traite des fourrures avant le passage de Lewis et Clark, était tombé dans les mains de J.-N. Nicollet. Trois quarts de siècle après la mort de ce dernier, le manuscrit demeurait ignoré dans ses papiers jusqu'au jour où le $\mathrm{D}^{\mathrm{r}}$ Abel l'y découvrit (University of Oklahoma Press, 1939). Edouard de Montulé, qui remonte le Mississipi et l'Ohio en bateau à vapeur, en rapporte quelques aperçus sur les colons français. Edward-D. Seeber a traduit et publié ses Travels in America, 1816-1817 (Indiana University Press, 1950). En 1840, Victor Tixier, un jeune Français, étudiant en médecine, visite les plantations françaises de la Louisiane, s'achemine à Saint-Louis, et, de là, jusque chez les Indiens Osages chez qui il vit plusieurs mois. Le récit des voyages et des observations de ce jeune homme publié par Albert-J. Salvan dans la French Review (8: 100-109, 1939), s'appuie sur sa traduction du Voyage aux Prairies Osages par Tixier (1844), annotée par moi et qu'a publiée l'University of Oklahoma Press en 1940.

Avant d'en venir aux études d'histoire sociale, j'attire l'attention sur quelques ouvrages de base, ouvrages inappréciables. Bien que collections de documents d'histoire politique avant tout, ils n'en contiennent pas moins une substance importante pour l'historien du social. L'importante série de documents du pays des Illinois commencée par Clarence Alvord et C.-E. Carter, dans les Illinois Historical Collections, a été continuée par feu Theodore-C. Pease. Pease publia en 1934 The French Foundations, 1680-1693, Anglo-French Boundary Disputes en 1936, Illinois on the Eve of the Seven Years War, 1747-1755 en 1940; en outre, il laissa à sa mort en 1949 un autre ouvrage prêt pour publication. Comme partie intégrante du rapport annuel de 1945 de l'American Historical Association, Lawrence Kinnaird publie (1946-1949) trois volumes qui ont pour titre: Spain in the Mississippi Valley, 1765-1794. Plusieurs de ces documents tirés 
de la collection Bancroft, ont trait à la partie occidentale du pays des Illinois. A.-P. Nasatir, un autre historien, a rédigé, pendant le dernier quart de siècle, plusieurs articles documentaires où il exploite les archives des Indes à Séville. En 1952 la St. Louis Historical Documents Foundation imprime son Before Lewis and Clark, deux volumes de documents qui décrivent l'exploration de la rivière Missouri de 1785 à 1804. Les tomes XVI et XVII des Territorial Papers of the United States par Clarence-E. Carter traitent du territoire des Illinois; et les tomes XIII, XIV et XV du territoire Missouri-Louisiane. Ces volumes, tous publiés depuis la guerre, renferment une quantité considérable de renseignements sur les Français de la vallée centrale du Mississipi. Un autre ouvrage documentaire peut être relevé ici où l'on apercevra l'emploi possible et profitable des registres publics et des papiers privés. Lors de la célébration du $250^{\circ}$ anniversaire de la fondation de Cahokia, la St. Louis Historical Documents Foundation a fait paraître, en mai 1949, Old Cahokia: $A$ Narrative and Documents Illustrating the First Century of its History. On y a présenté un index de documents relatifs à l'histoire de l'église catholique, un choix judicieux, dans les registres officiels, de conventions d'associés, de procès, de contrats de mariage, de testaments, de procédures d'homologation, d'inventaires de succession, d'actes de décès, d'une tranche considérable de la correspondance d'un homme d'affaires, de lettres afférentes à la prise de Cahokia par les Américains en 1778, enfin le dossier de deux causes judiciaires de portée historique: le tout précédé d'une notice sur Cahokia et ses habitants.

Sur la vie des établissements des Illinois, en tant que groupe historique, le professeur Carrière a fourni, il y a une décennie et demie, deux contributions remarquables par la quantité de matière qu'il a su entasser en peu d'espace: l'une sur les «Life and Customs in the French Villages of the Old Illinois Country, 1763-1939 », faisait partie du rapport annuel de la Société historique du Canada pour 1939 (pp. 34-47) ; l'autre, parue dans le Canada Français (28: 457-76, 1941) traitait de «L'Oeuvre de la France dans la vallée du Mississipi ». Notons un autre travail intéressant sur les premiers établissements: «The French 
Villages of the Illinois Country » par Natalia-M. Belting, étude parue en 1943 dans la Canadian Historical Review (24:14-23).

Le $250^{\circ}$ anniversaire de Cahokia a fait surgir quantité d'articles sur l'histoire de cette petite ville. Comptons parmi les plus précieux, un long travail d'un ingénieur en architecture, Charles-E. Peterson, du service des parcs nationaux. L'œuvre porte un titre modeste, en dépit de sa perfection. Publiées d'abord en 1949 dans la French American Review, "Notes on Cahokia » ont été reproduites, quelque peu augmentées, en trois numéros de l'Illinois State Historical Society Quarterly. Kaskaskia a été également heureuse dans l'attention qui lui fut accordée. En 1945, Mlle Belting a publié dans l'Indiana Magazine of History (41: 1-18) son «Kaskaskia: 'The Versailles of the West' »; trois ans plus tard, elle y ajoutait sa monographie sur Kaskaskia under the French Régime (University of Illinois Press), étude qui, comme celle de Peterson, avait largement mis à profit des sources inexploitées et, pour la première fois, projeté beaucoup de menues connaissances sur les soixante première années de cette ville. Mise en vedette par la célébration de son bicentenaire, en 1935, Sainte-Geneviève gagna d'obtenir son histoire, en cent cinquante pages, par Francis-J. Yealy, s.j. En 1941 Peterson donna à la Missouri Historical Review (35: 207-32) un travail important sur «Early Ste. Genevieve and its Architecture ».

Des récentes études sur Saint-Louis, cinq mériteraient mention. Feu James-B. Musick, en ce temps-là secrétaire du City Art Museum de Saint-Louis (Nous, Franco-Américains, sommes un groupe fort mêlé) a publié, en 1941, une monographie de St. Louis as a Fortified Town. Préoccupé uniquement de cet aspect particulier de l'histoire coloniale, Musick nous a donné là une étude peut-être définitive. Comme principal apport de Peterson à l'histoire de Saint-Louis, citons son savant Colonial St. Louis: Building a Creole Capital. Cette étude, parue d'abord en partie dans le Missouri Historical Society Bulletin, a plus tard été mise en volume par la Société (1949). Peterson ne s'était principalement proposé que d'écrire l'histoire de l'architecture à Saint-Louis. Il n'en a pas moins rassemblé une somme considérable de menus faits, tirés surtout d'archives locales inédites, 
sur les métiers de la construction et sur la physionomie de la ville. Rangeons dans une autre catégorie mon Private Libraries in Creole Saint Louis édité en 1938 par l'Institut français de Washington. On y trouve un aperçu des niveaux de vie et de l'état de la culture intellectuelle, en tête d'une recension détaillée des possesseurs de livres tirée de sources authentiques de l'époque coloniale et du début de l'ère américaine. Un autre de mes travaux qui, je crois, mérite mention, c'est «Louis-R. Cortambert and French Newspapers in St. Louis, 1809-1854 », paru dans les Papers of the Bibliographical Society of America en 1941. Parmi les récentes publications sur Saint-Louis, signalons l'ouvrage de la St. Louis Historical Documents Foundation sur The Early Histories of St. Louis (1952) qui offre sept récits authentiques précédés d'un essai historiographique.

Pour le Missouri en dehors de Saint-Louis, retenons, en particulier, trois travaux. En 1930, Ovid Bell a publié une petite histoire de la Côte Sans Dessein. Harvey Wish a donné à MidAmerica (23: 167-189, 1941) un travail sur «The French of Old Missouri (1804-1821), a Study in Assimilation »; Ernest-J. Liljegren, un article remarquable sur la «French Education in Spanish Louisiana » à la Missouri Historical Review (35: 345$72,1941)$, étude élaborée à même des pièces des Archives des Indes.

Une autre phase de la vie culturelle a été, ces derniers temps, l'objet d'étude. Dans The French in America, 1520-1880, catalogue d'une superbe exposition au Detroit Institute of Arts, 1951, Paul Grigaut a fait connaître les arts du pays illinois. Deux autres catalogues pour deux expositions au City Art Museum de Saint-Louis, Mississippi Panorama (1949) et Westward the Way (1954), toutes deux organisées par Perry $\mathrm{T}$. Rathbone, ont de quoi illustrer la vie des Français de la vallée du Mississipi. Notons encore quelques études spéciales, par exemple, la découverte, par Charles Van Ravenswaay, des esquisses de Maria von Phul qui peignent les mœurs et coutumes de Saint-Louis vers 1818 ( Missouri Historical Society Bulletin, $10: 367-84,1954)$; aussi mes articles sur Léon Pomarède dans le 
Bulletin of the City Art Museum of St. Louis (1949) et dans le Bulletin de l'Institut français de Washington (1952). Apport également d'un grand intérêt que le compte rendu et commentaire de R. W. G. Vail, « The American Sketchbooks of CharlesAlexandre Lesueur, 1816-1837 (American Antiquarian Society Proceedings, 1938). André Maury étudie dans la French American Review (1:161-73, 1948), l'œuvre de ce peintre naturaliste, et Gilbert Chinard, le plus vigoureux et le plus fécond des lettrés franco-américains, en a donné une autre étude aux Proceedings de la American Philosophical Society (93: 114-18, 1949).

Un grand nombre d'autres livres et travaux à la fois intéressants et valables, ont vu le jour en ce dernier quart de siècle. Je ne puis qu'en donner une brève énumération qui en montrera la variété: "The French in Early Kentucky par Hentley Dupré (Filson Club Quarterly, 15: 78-104, 1941) ; Education and Reform at New Harmony - the Correspondence of William Maclure and Marie Duclos Fretageot, 1820-1833 par Arthur-E. Bestor (Indiana Historical Society, 1948) ; «The Bruté Library in Vincennes » par Edward-D. Seeber (Indiana Quarterly for Bookmen, 14: 81-86, 1948) ; Barthélemi Tardiveau: A French Trader in the West, par Howard-C. Rice (Institut français de Washington, 1938); L'odyssée américaine d'une famille française, le docteur Antoine Saugrain, par Mme Fouré-Selter (Institut français, 1936) ; The Jesuit Missions in the Illinois Country (1673-1763) (1933), par Sœur M.-Borgia Palm; Chapters in Frontier History par Gilbert J. Garraghan (Milwaukee, 1934); The Society of the Sacred Heart in North America, par Mère Louise Callan (New York, 1937). Notice de la même sur l'abbé Gibault dans le Missouri Historical Society Bulletin (5: 26688, 1949); The French in the Mississippi Valley, 1740-1750 par Norman Caldwell (University of Illinois Press, 1941) ; Pontiac and the Indian Uprising, par Howard Peckham (Princeton University Press, 1947) ; "Huet de la Valinière », par Thomas-F. Cleary (Mid-America, 15: 213-28, 1933) ; "Pierre Menard of Kaskaskia », par William-S. Merrill (Mid-America, 14: 15-18, $1931)$; le «Missouri Reader», articles qui résument l'activité française en la région (Missouri Historical Review, 1945-47); 
The Greatest Real Estate Deal in History: the Louisiana Purchase - 1803, par John-Francis Bannon et quatre membres de sa section d'histoire à l'université de Saint-Louis (Saint-Louis, 1953). Qu'une telle liste puisse s'accorder de considérables allongements, il n'est que d'ouvrir «Anglo-French and France-American Studies: A current Bibliography » dirigé par Donald-F. Bond de 1938 à 1947, et depuis lors par Joseph-M. Carrière.

On tient là le rapport succinct de ce qui a été écrit, dans le dernier quart de siècle, sur l'histoire culturelle de la région illinoise. $\mathrm{Du}$ travail actuellement en cours, nous pourrions risquer un mot, quoique, en grande partie, sans doute, il ait échappé à notre connaissance. Frederick-A. Palmer prépare une étude des Westerners at Home - Society in the Middle West, 1800-1840, as seen by French and British Travellers; Allen-J. Barthold élabore, quant à lui, une History of the French Press in America; Charles van Ravenswaay, directeur de la Missouri Historical Society, est en train d'écrire une histoire de SaintLouis. Mère Louise Callan s'occupe à une vie de Mère Duchesne, fondatrice des «dames » du Sacré-Cœur en Amérique et Father Rahill, à une nouvelle histoire de l'archidiocèse de Saint-Louis. Marcel Giraud, titulaire de la chaire d'histoire de l'Amérique du Nord au collège de France, a déjà effleuré Cahokia dans son Histoire de la Louisiane française: le règne de Louis XIV (Paris, 1953); dans ses prochains volumes les Illinois feront l'objet d'une étude plus poussée. Lawrence Kinnaird tient deux autres volumes de son Spain in the Mississippi Valley: 1794-1804 prêts pour publication. Colton Storm est à éditer une collection des lettres du lieutenant-colonel John Wilkins écrites pendant les trois années de son commandement au pays des Illinois, à l'époque de 1760. Le professeur Carrière complète, en ce moment, une édition des fameuses lettres de Charlevoix sur la vallée du Mississipi ; le professeur Chinard publiera le texte intégral de la narration de Dumont de Montigny que l'on ne peut se procurer que sous la forme abrégée parue il y a deux cents ans. A.-P. Nasatir a traduit cinq journaux de bord des canonnières espa- 
gnoles sur le Mississipi dans les années 1790, journaux qui fournissent une image intéressante de «life on the Spanish Mississippi ». Le Père Joseph-P. Donnelley de l'université Creighton (autrefois de l'université Saint-Louis) a mis en anglais les notes de voyage manuscrites du Père Nicolas Point qui se rendit aux Montagnes Rocheuses avec de Smet dans les années 1840. Ces manuscrits d'un artiste amateur qui, au cours de ses voyages, a rempli ses cahiers de croquis, offrent un particulier intérêt. Cet ouvrage sera publié par la St. Louis Historical Documents Foundation. Moi-même, je prépare deux ouvrages documentaires: l'un qui sera une collection de lettres de J.-N. Nicollet; l'autre contiendra des lettres d'Audubon à sa famille, lors de son voyage sur le Missouri en 1843. Une bonne partie des lettres d'Audubon ont trait à Saint-Louis. L'Université Laval se propose de publier bientôt le journal et la description du Missouri de Jean-Baptiste Trudeau écrits dans les années 1790 et connus jusqu'à présent que de manière fragmentaire. Georges-J. Joyaux travaille à une bibliographie des journaux de langue française.

En dépit de ce grand nombre d'études franco-américaines en préparation, il va de soi qu'il reste encore beaucoup à faire. Au sujet, par exemple, de la colonie icarienne qui occupa Nauvoo après le départ des mormons, toute une somme de riches matériaux n'a jamais été traduite ni même abordée par le chercheur. Les papiers de Gallatin à la New York Historical Society contiennent une fort belle collection de lettres adressées de Vincennes, par Jean Badollet à Gallatin, qui constitueraient un ouvrage documentaire d'un très vif intérêt. Il en serait de même pour les lettres de Jean-B.-C. Lucas qui émigra de Normandie en Pennsylvanie en 1784 et qui mourut à Saint-Louis en 1842: des tas de sa correspondance, tant publique que privée, sont à la New York Historical Society, aux Archives nationales américaines et à la Missouri Historical Society. Il y aurait lieu, à coup sûr, de pousser l'étude de la vie française à Vincennes. Une enquête sur les infiltrations de la pensée révolutionnaire française dans les deux parties des Illinois mériterait d'être entreprise, de même qu'une étude du fonctionnement du gouvernement régional. Le journal inédit de J.-N. Nicollet, actuelle- 
ment à la bibliothèque du Congrès, et les lettres de Charles Gratiot qui sont à la Missouri Historical Society devraient être éditées. Une bibliographie de la littérature de voyage dans les journaux et périodiques français serait extraordinairement utile.

Encore ne sont-ce là que quelques-uns des nombreux projets qui viennent à l'esprit. Une recherche menée scientifiquement dans les masses de documents officiels à Saint-Louis, Springfield, Urbana, Chicago, Washington, Détroit, Montréal, Québec, dans les registres des bureaux d'enregistrement et cours régionales, dans les journaux des premiers temps, une recherche et une exploration des collections de lettres et journaux personnels, révèleraient, à n'en pas douter, maints documents d'un riche intérêt pour le pays des Illinois.

John Francis MCDeRmotT,

Associate Professor of English,

Washington University, U.S.A.

\section{Comme quoi l'on en veut encore à l'histoire scientifique}

(Suite de la page 501)

Faguet ne lisait que lui pour s'informer des origines de la France contemporaine. C'est l'art d'Henri Houssaye, l'art de Sorel, qui les font survivre à l'impeccable Charles Seignobos. Je pense qu'un jour «La Pléiade » accueillera sur son papier-bible Fustel, Sorel et Houssaye. Mais non la foule des exacts et des ennuyeux. Si je blasphème, que Clio me pardonne. Je sais qu'elle ne pardonne pas aisément.

«Voici M. de Madariaga qui, lui aussi, brille d'art, d'enthousiasme, d'ironie, de hardiesse provocante. Autant de défauts, aux yeux des purs et de mérites pour la postérité. Après l'histoire de Colomb, dont je vous ai dit naguère les beautés, et celle de Fernand Cortès, il nous donne celle de Bolivar, telle qu'il la voit, tel qu'il la «sent». Le souci de construire une trilogie est un souci d'architecte. Numero deus impare gaudet. Il touche, en passant, San Martin, dont Bolivar aperçut, par-dessus les Andes, la silhouette fraternelle. Mais il n'a pas besoin d'un quatrième. Décidément il est poète.

«Que va-t-il nous dire de Bolivar ? Que ce libérateur n'a pas donné la liberté, du moins une véritable liberté, aux nations qu'il a enfantées. D'après M. de Madariaga, elles n'en avaient guère besoin...»

Robert Kemp, La Vie des livres. Désunion d'un monde. Les Nouvelles littéraires [Paris] 19-1-1956.

(Découpure fournie par M. Jean-Jacques LEFEBVRE) 